\title{
Assessing the therapeutic potential of APPs $\alpha$ in a tau transgenic mouse model of Alzheimer's disease
}

Charlotte Bold ${ }^{1}$, Danny Baltissen ${ }^{1}$, Susann Ludewig ${ }^{2}$, Roman Spilger $^{3}$, Karl Rohr ${ }^{3}$, Martin Korte ${ }^{2}$, Ulrike Müller ${ }^{1}$

${ }^{1}$ Institute for Pharmacy and Molecular Biotechnology (IPMB), Heidelberg, ${ }^{2}$ Cellular Neurobiology, Braunschweig, ${ }^{3}$ BIOQUANT Center, Heidelberg A large body of evidence indicates a neuroprotective and neurotrophic function for APPs $\alpha$ not only in vitro, but also when expressed by AAV vectors in vivo such as in APP/PS1 transgenic AD model mice with $A \beta$-induced pathology [1,2,3]. Previously, we could show that $A P P s \alpha$ rescued deficits of APP/PS1 in synaptic plasticity and spine density and also reduced plaque deposition [3]. Thus, it is crucial to test a more general applicability of APPs $\alpha$ as a treatment for AD and to assess whether APPs $\alpha$ is also beneficial in mice with tau-induced pathology.

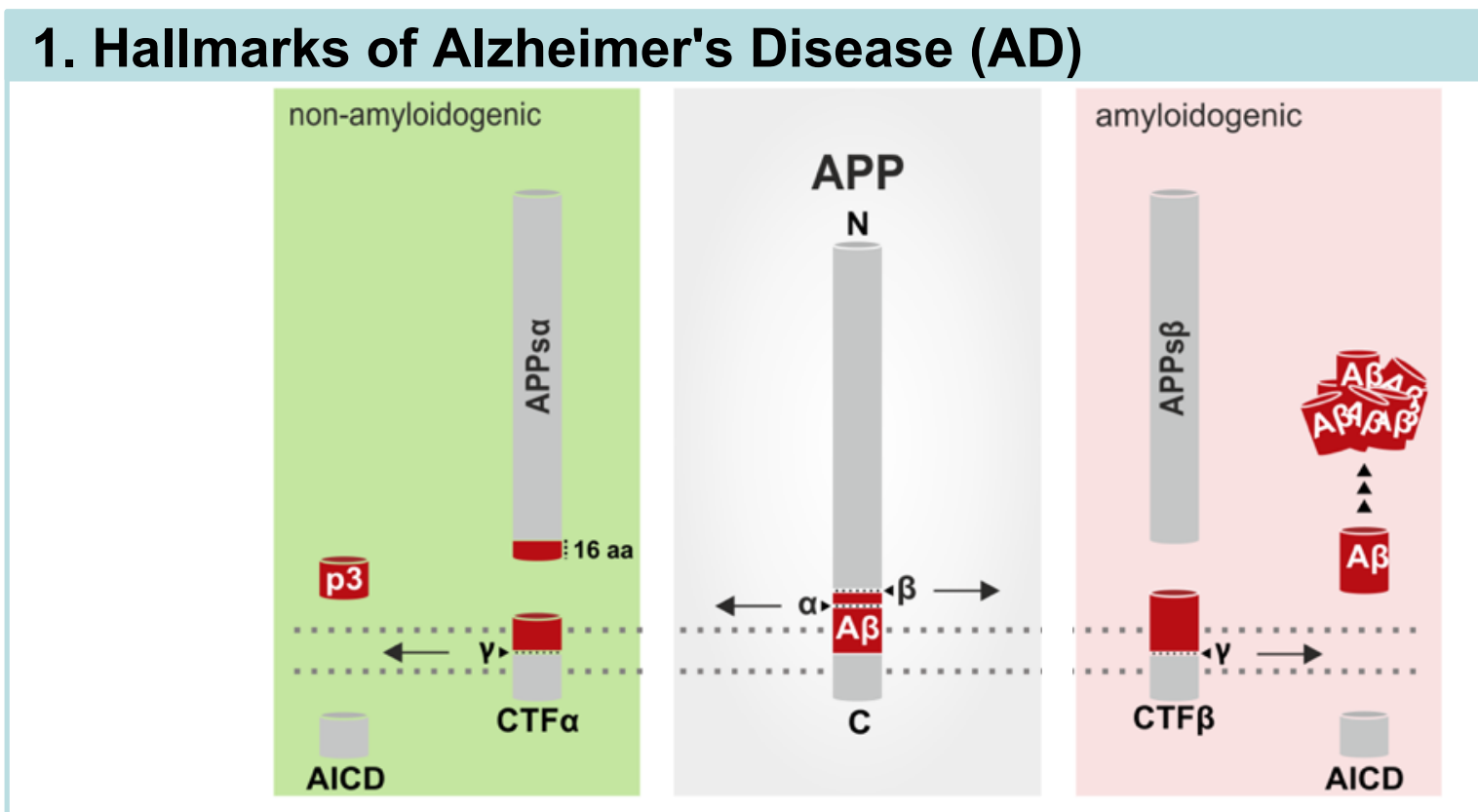

$A D$ is the most prevalent neurodegenerative disease in the elderly, characterized by two major pathological hallmarks: senile $A \beta$ plaques and neurofibrillary tau tangles ${ }^{[4]}$. The amyloid precursor protein (APP) can be cleaved in two major competing pathways: amyloidogenic and non-amyloidogenic processing, either giving rise to APPs $\alpha$ or $A \beta{ }^{[5]}$.
2. Mutation and pathology in the tau transgenic mouse line P301S

hTau.P301S mouse line

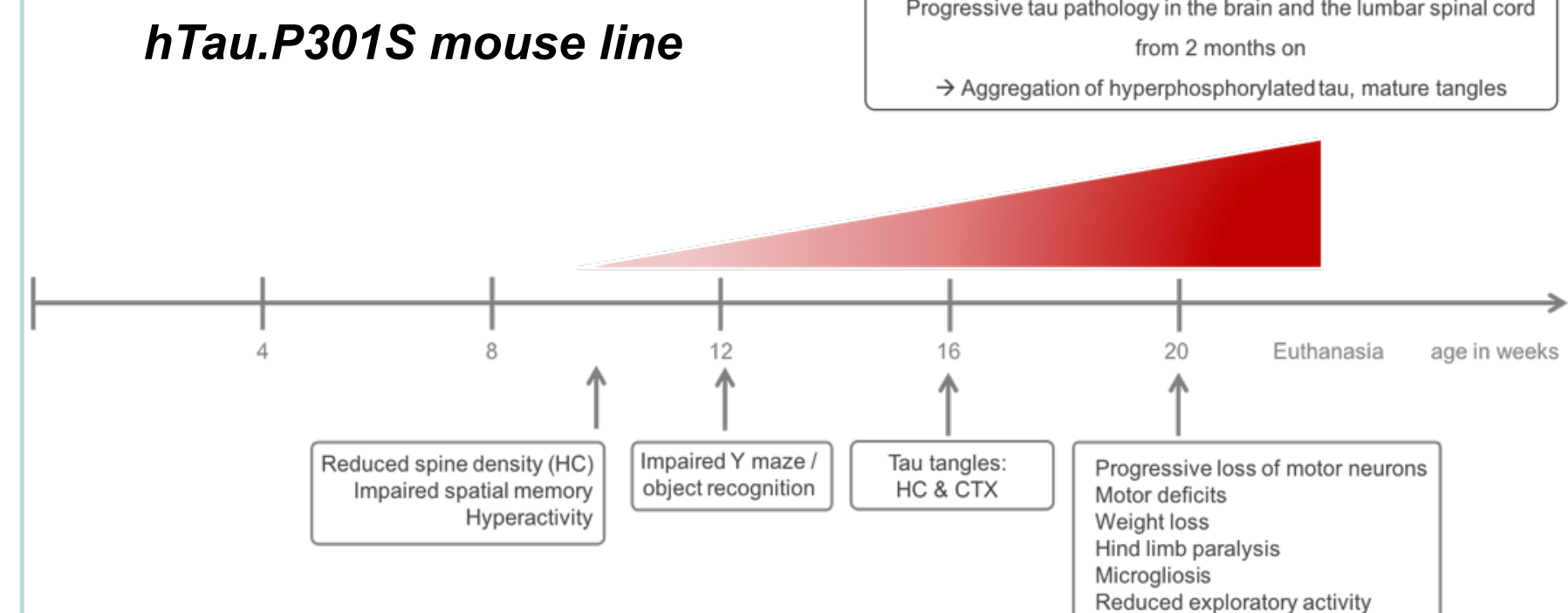

The hTau.P301S AD mouse model expresses a 383 aa human tau isoform harboring a mutation at residue P301S. First cognitive deficits are correlated with reduced spine density (10 weeks). At later stages, mature tau tangles are present in hippocampus and cortex ${ }^{[6]}$.

3. Increased LTP in P301S mice is accompanied by the loss of GABAergic interneurons in the hippocampus

A

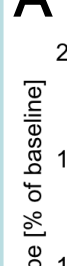
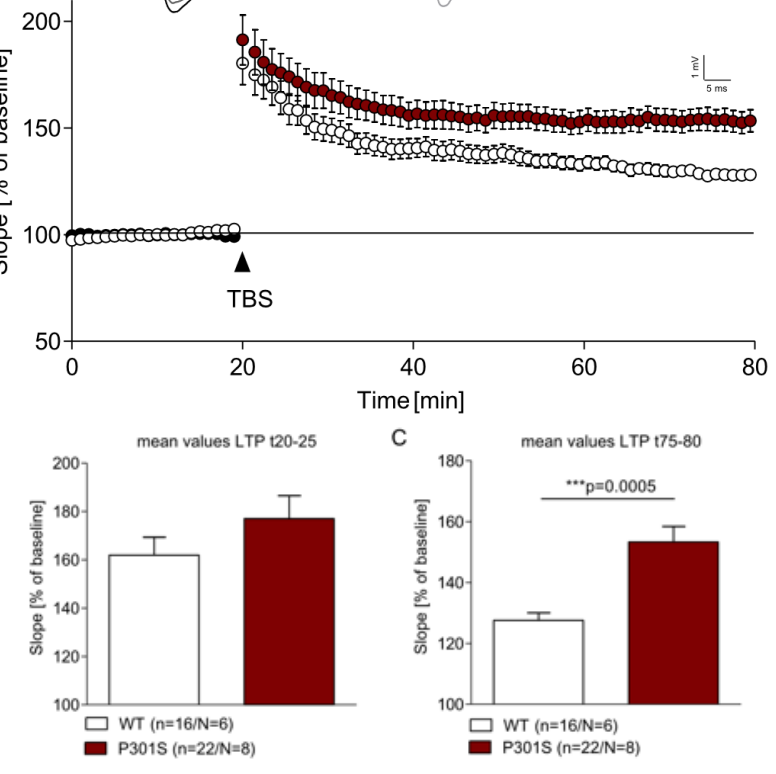

B WT, 16-18 weeks
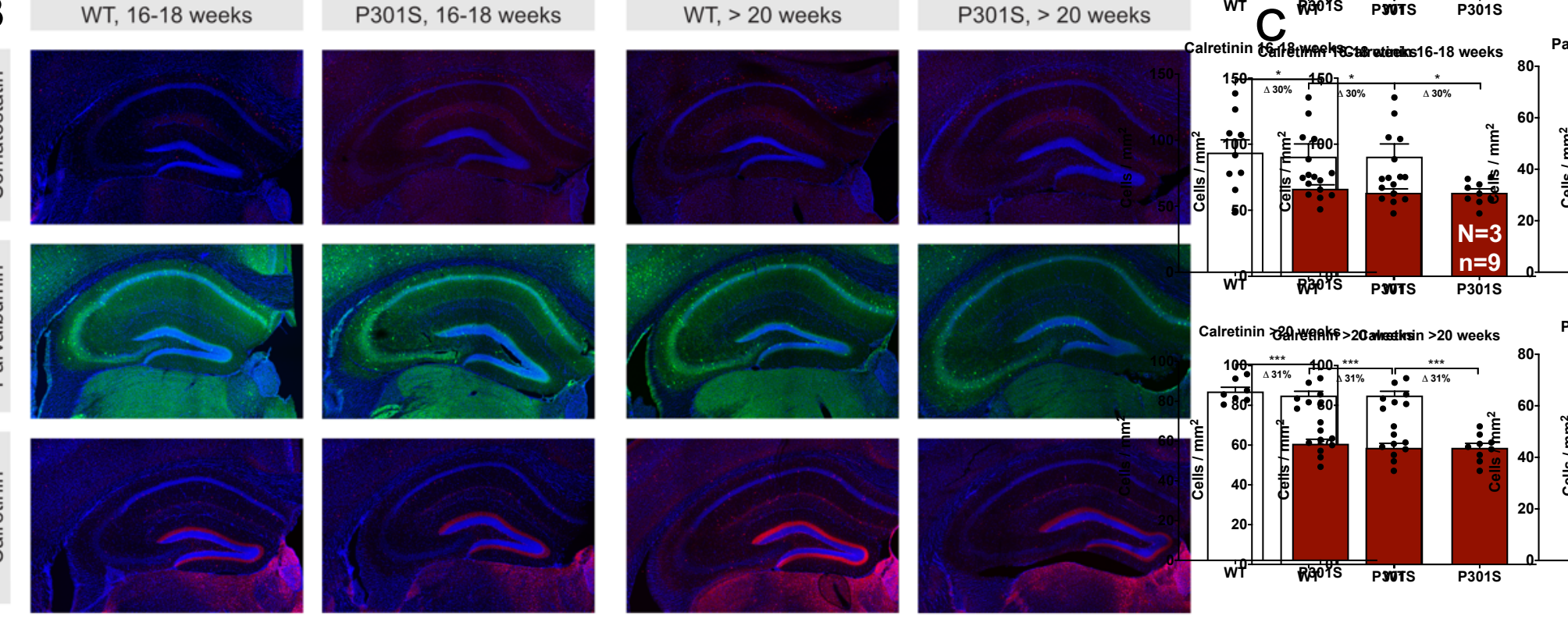

A Activity-dependent synaptic plasticity was investigated on acute hippocampal slices of 16-18 weeks old WT and P301S mice. LTP measurement revealed a significant increase in LTP maintenance in P301S mice. B + C The elevated LTP is accompanied by a significant loss of different GABAergic interneuron subpopulations in the hippocampus (Calretinin-, Parvalbumin- and Somatostatin-positive cells). Reduced inhibitory input might explain the increased LTP in P301S mice. A similar phenotype is published for the JNPL3 tau transgenic mouse line [7].

\section{Beneficial effects of APPs $\alpha$ in an AD mouse model}

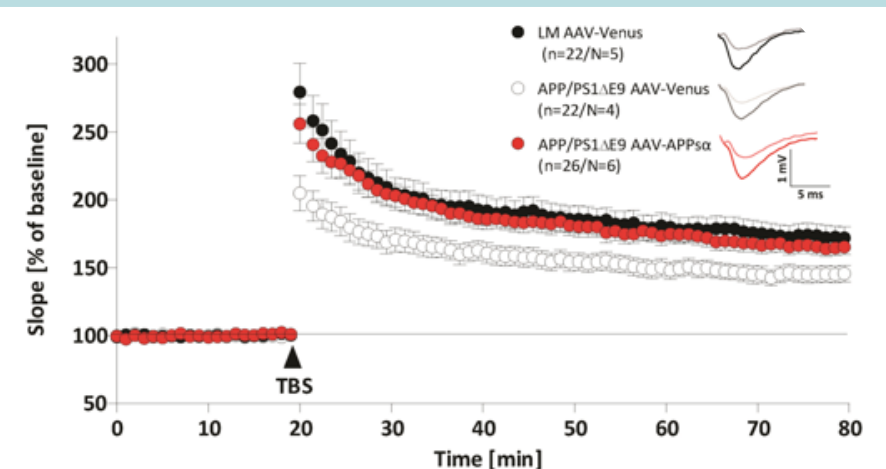

\section{Elevated LTP in P301S is normalized to WT level by recAPPs $\alpha$}
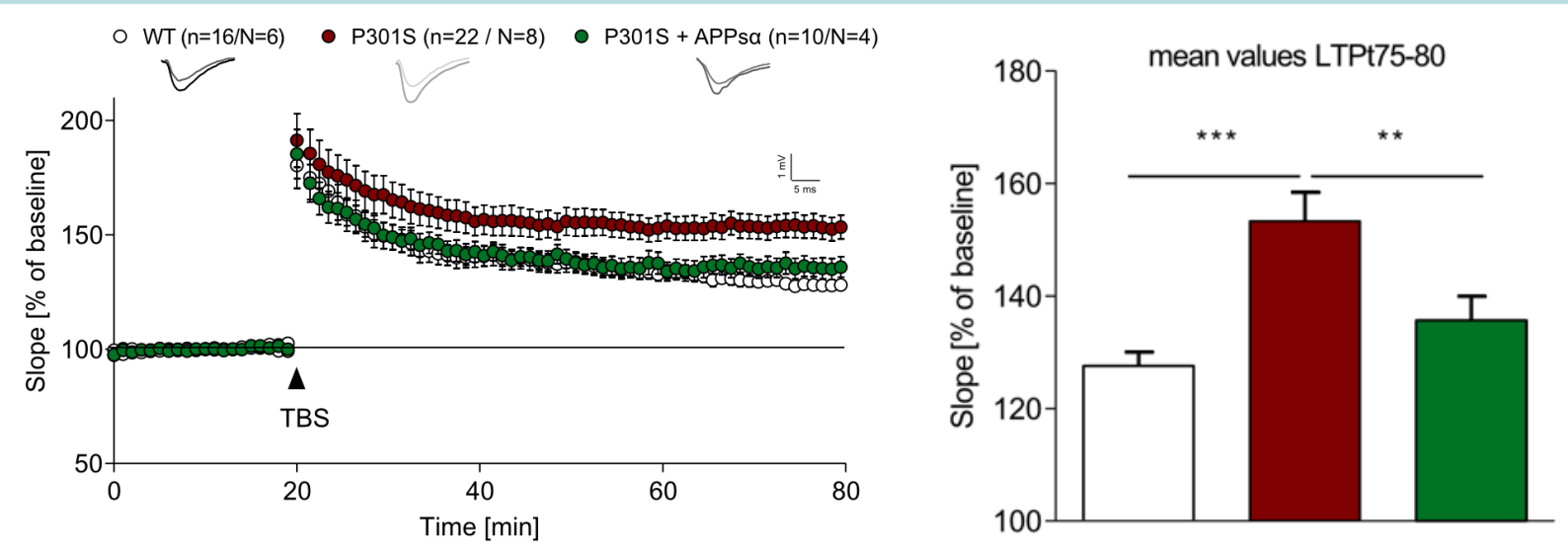

LTP was investigated on acute hippocampal slices of 16-18 weeks old WT, P301S and P301S mice with APPsa incubation which normalized enhanced LTP in P301S mice to WT level. Does AAV-mediated application of APPs $\alpha$ have beneficial effects in P301S in vivo? with preexisting plaque pathology restored deficits in synaptic plasticity, spine density, spatial memory and lead to a reduced plaque load [3]. Can functional deficits in P301S mice be rescued by APPs $\alpha$ application in vitro?

\section{AAV-APPs $\alpha$ rescues the spine density deficit in P301S mice}

\begin{tabular}{|c|c|}
\hline $\begin{array}{l}\text { stereotactic injection of } \\
\text { AAV-APPsa or AAV-Venus }\end{array}$ & Analysis \\
\hline 1 & \\
\hline 6 & \\
\hline
\end{tabular}

B ITR - Syn

sin - Venus - ITR

A + B At the age of 6 weeks, P301S and WT mice were intracranially injected with either AAV-Venus (control vector) or AAV-APPs $\alpha$. Analysis was conducted at the age of either 16 or 20 weeks. C The spine density of CA1 neurons in P301S mice was significantly reduced in midapical and basal dendrites at both investigated time points and rescued to WT level by the overexpression of

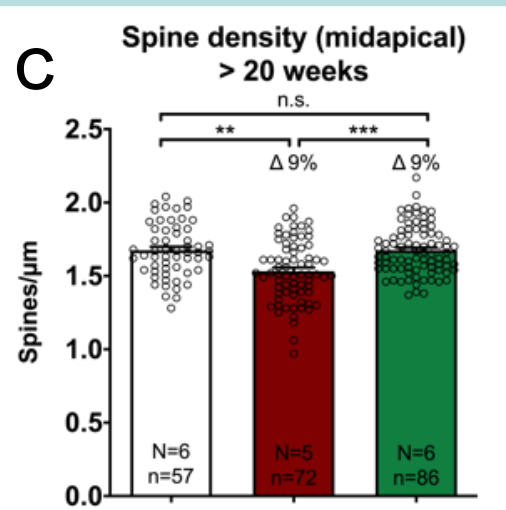

Spine density (midapical) $16-18$ weeks

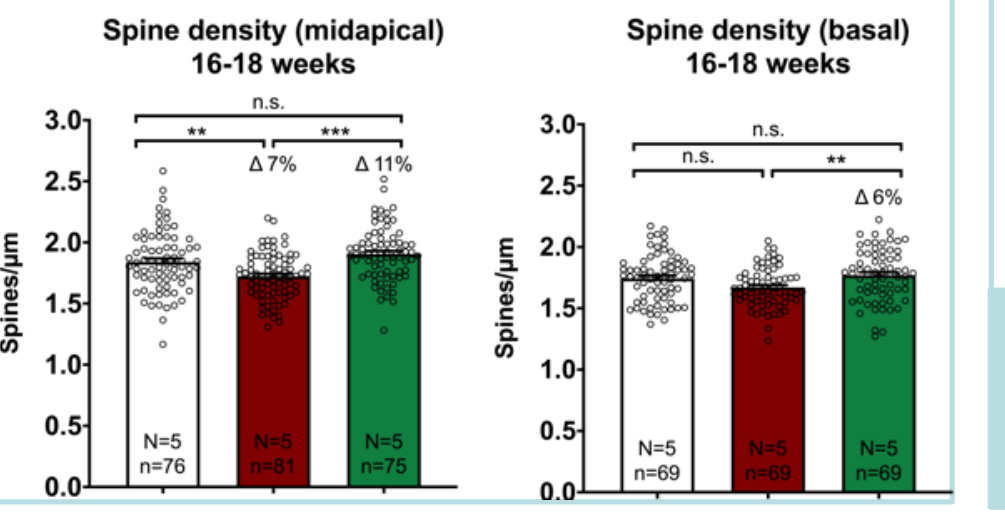

\section{Summary and outlook}

- Loss of inhibitory interneurons in the HC

- Application of recAPPsa normalizes elevated LTP to WT level

- AAV-APPs $\alpha$ injection rescues spine density deficit in CA1 neurons

- Hypothesis: Are early effects in P301S mice caused by the interneuron deficit rather than aggregated tau species?

References: [1] Hick et al., 2015 [2] Richter et al., 2018 [3] Fol et al., 2016 [4] Jucker and Walker, 2013 [5] Müller, Deller, Korte, 2018 [6] Allen et al., 2002 [7] Levenga et al., 2013

Contact: u.mueller@urz.uni-hd.de, bold@uni-heidelberg.de DFG grant: MU 1457/14-1 